\title{
Security Analysis of the Certificateless Signature Scheme Proposed at SecUbiq 2006
}

\author{
Je Hong Park ${ }^{1}$ and Bo Gyeong Kang ${ }^{2}$ \\ ${ }^{1}$ ETRI Network \& Communication Security Division \\ jhpark@ensec.re.kr \\ 2 Samsung Electronics Co., LTD \\ bogyeong. kang@samsung.com
}

\begin{abstract}
In this paper, we show that the certificateless signature scheme proposed by Yap, Heng and Goi at SecUbiq 2006 is insecure against a key replacement attack and a malicious-but-passive KGC attack, respectively. The former implies that anyone who replaces a signer's public key can forge valid signatures for that signer without knowledge of the signer's private key. The latter supposes the malicious-but-passive KGC, which generates system parameters based on the information of the target user to impersonate. Our results are based on the fact that the private key of the YHG scheme has the form of a BLS multisignature generated by the KGC and the user. Finally, we review the vulnerability of several certificateless signature schemes under theses attacks.
\end{abstract}

\section{Introduction}

The certificateless cryptosystem introduced by Al-Riyami and Paterson [1] is designed to overcome the key escrow limitation which is inherent in identitybased cryptosystems. Each user has a unique identifier, and a semi-trusted third party called the Key Generation Center(KGC) generates the partial private key associated with that identifier using its own master secret key and sends it to the user with that identifier. But the user also holds a secret value which is chosen by him/herself, and the user combines the partial private key with the secret value to generate his/her actual private key. That is, the user's private key is not generated by the KGC alone and so the KGC does not know the user's private key that implies the escrow freeness. Independent to the identifier, the user also publishes the public key, based on the secret value and system parameters. Note that the user's public key does not need to be certified by any trusted authority as in conventional PKIs. The structure of the certificateless scheme ensures that the key can be verified without a certificate. So its security model supposes the adversary who may attempt to replace a user's public key with a value of its own choice. This is called in general a key replacement attack 94 4,667] and, successfully applied to some certificateless signature schemes such as [1,5].

In the original security model for certificateless cryptosystems [1, the KGC generates its master public/private key pair honestly, according to the scheme specification. However, the modified security model proposed by $\mathrm{Au}$ et al. 2] 
removes this assumption. So the user's trust on the KGC is further relaxed. In detail, the KGC may not follow the scheme specification for generating system parameters and master key, while it does not actively replace a user's public key or corrupt the user's private key. The purpose of such a KGC is to compromise the target user's private key without being detected. Note that the malicious $\mathrm{KGC}$ is still passive, in the sense that the KGC would not actively replace the user public key or corrupt the user private key. It was shown in [2] that Al-Riyami-Paterson scheme [1] and Huang-Susilo-Mu-Zhang scheme 9] are vulnerable in this security model.

In SecUbiq 2006, Yap, Heng and Goi proposed a certificateless signature scheme (called the YHG scheme here) and claimed that their scheme is efficient, comparison to previous schemes [15]. Their improvement is supported by the lack of public key validation which requires pairing computations in the signature verification phase. We, however, show that the YHG scheme is insecure against a key replacement attack and a malicious-but-passive KGC attack, respectively. The former attack is based on the fact that the user private key of the YHG scheme has the form of a BLS multisignature [3] generated by the KGC and the user. We will apply a rogue attack for BLS multisignatures to the YHG scheme: Due to the lack of public key validation in the signature verification phase, a verifier cannot ensure that the signer knows the secret value. It implies that an adversary who replaces a signer's public key can forge signatures of that signer, without knowledge of the signer's private key. Additionally, we show that the malicious KGC can generate master public key using the target user's identifier and so it may impersonate that user easily, as described in [2]. Note that the Gorantla-Saxena scheme [5] and its improved scheme [17] are also vulnerable to this attack, due to structural similarity.

This paper is organized as the follows. We briefly review the YHG scheme in Section 2, and then analyze its security against two types of attacks in Section 3 . We conclude in Section 4

\section{Review of YHG Certificateless Signature Scheme}

Throughout this paper, $\left(\mathbb{G}_{1},+\right)$ and $\left(\mathbb{G}_{2}, \cdot\right)$ denote two cyclic groups of prime order $q$. A pairing is an efficiently computable, non-degenerate function $e: \mathbb{G}_{1} \times$ $\mathbb{G}_{1} \rightarrow \mathbb{G}_{2}$ with the bilinearity property that $e(P+Q, R)=e(P, R) \cdot e(Q, R)$ and $e(P, Q+R)=e(P, Q) \cdot e(P, R)$ for $P, Q, R \in \mathbb{G}_{1}$. Let $H_{1}:\{0,1\}^{*} \rightarrow \mathbb{G}_{1}$ and $H_{2}:\{0,1\}^{*} \times \mathbb{G}_{1} \rightarrow \mathbb{Z}_{q}^{*}$ be hash functions. These are used as a part of the system parameters generated by the KGC. The YHG certificateless signature scheme can be described as follows:

- Setup: Given a security parameter $k$, the KGC chooses an arbitrary generator $P \in \mathbb{G}_{1}$, selects a random $s \in \mathbb{Z}_{q}^{*}$ and sets $P_{0}=s P$. Then the system parameters are params $=\left\langle\mathbb{G}_{1}, \mathbb{G}_{2}, e, q, P, P_{0}, H_{1}, H_{2}\right\rangle$. The message space is $\mathcal{M}=\{0,1\}^{*}$. The master secret key is $\mathrm{mk}=s$. 
- Set Partial Private Key: Given the system parameters params, the master secret key mk and a user $A$ 's identifier $\mathrm{ID}_{A}$, the KGC computes $Q_{A}=$ $H_{1}\left(\mathrm{ID}_{A}\right) \in \mathbb{G}_{1}$ and outputs the partial private key $D_{A}=s Q_{A}$.

- Set Secret Value: Given the system parameters params, the user $A$ selects a random value $x_{A} \in \mathbb{Z}_{q}^{*}$ as the user secret value.

- Set Private Key: Given the system parameters params and the partial private key $D_{A}$, the user $A$ computes the user private key $S_{A}=x_{A} Q_{A}+D_{A}$.

- Set Public Key: Given the system parameters params and the secret value $x_{A}$, the user $A$ computes the user public key $P_{A}=x_{A} P \in \mathbb{G}_{1}$.

- Signature Generation: Given the system parameters params, the identifier $\mathrm{ID}_{A}$, a message $m \in \mathcal{M}$ and the private key $S_{A}$, the user $A$ randomly chooses $r \in \mathbb{Z}_{q}^{*}$ and sets $U=r Q_{A} \in \mathbb{G}_{1}$. Then computes a signature $\sigma=(U, V)$ for the message $m$ where $V=(r+h) S_{A}$ and $h=H_{2}(m, U)$.

- Signature Verification: Given a signature/message pair $(\sigma, m)$, the signer's identifier $\mathrm{ID}_{A}$ and the signer's public key $P_{A}$, the verifier computes $h=$ $H_{2}(m, U)$ and checks whether $e(P, V)=e\left(P_{0}+P_{A}, U+h Q_{A}\right)$. If not, then rejects the signature else accepts it as valid.

The authors claim that this scheme is provably secure and more efficient then previously proposed schemes because fewer bilinear pairing computations are required [15]. As described above, this scheme requires only two pairing computations in the signature verification phase. This efficiency is induced from the lack of public key validation. We will show that this fact makes the YHG scheme vulnerable to the key replacement attack.

\section{Security Analysis}

\subsection{Key Replacement Attack}

Without loss of generality, the signer forwards his/her public key to the intended verifier(s) and announces his/her identifier. So an adversary who wants to forge a signature of a user $A$ with the identifier $\mathrm{ID}_{A}$ runs as follows:

1. Randomly chooses $x \in \mathbb{Z}_{q}^{*}$ and computes a signature $\sigma=(U, V)$ for a message $m \in \mathcal{M}$ as follows:

$$
U=r Q_{A}, h=H_{2}(m, U) \text { and } V=(r+h) x Q_{A},
$$

where $Q_{A}=H_{1}\left(\mathrm{ID}_{A}\right)$ and $r \in \mathbb{Z}_{q}^{*}$.

2. Sets $P_{A}^{\prime}=x P-P_{0}$ as a public key of the user $A$.

3. Then sends the signature $\sigma$, the message $m$, the identifier $\operatorname{ID}_{A}$ and the public key $P_{A}^{\prime}$ to the verifier(s).

Then the verifier computes $h=H_{2}(m, U)$ and checks whether $\left\langle P, P_{0}+P_{A}^{\prime}, U+\right.$ $\left.h Q_{A}, V\right\rangle$ is a valid Diffie-Hellman tuple. Since $e(P, V)=e\left(P,(r+h) x Q_{A}\right)$ and $e\left(P_{0}+P_{A}^{\prime}, U+h Q_{A}\right)=e\left(x P,(r+h) Q_{A}\right), e(P, V)=e\left(P_{0}+P_{A}^{\prime}, U+h Q_{A}\right)$ and so $\left\langle P, P_{0}+P_{A}^{\prime}, U+h Q_{A}, V\right\rangle$ is valid. Hence $\sigma$ is verified as a valid signature for the message $m$ generated by the user $A$. 
This attack is based on the algebraic structure of a user's private key in the YHG scheme. Since the signer $A$ 's private key $S_{A}$ has the form of a BLS multisignature 3 generated by the $\mathrm{KGC}$ and the signer $A$, we can apply a rogue attack using the key substitution trick. In general, a rogue attack for BLS multisignatures can be described as follows: Let $p k_{A}=x_{A} P$ and $p k_{B}=x_{B} P$ be two public keys of the user Alice and Bob, respectively. But Bob replaces $p k_{B}$ by $p k_{B}-p k_{A}$. Then for a message $m, x_{B} H_{1}(m)=x_{A} H_{1}(m)+\left(x_{B}-x_{A}\right) H_{1}(m)$ can be regarded as a valid multisignature on $m$ by both Alice and Bob. In the YHG scheme, the KGC plays the role of a honest user to generate a multisignature for the identifier $\operatorname{ID}_{A}$ of a user $A$ and is prohibited to replace the user $A$ 's public key. But a third party can use this key substitution trick for BLS multisignatures to forge a YHG signature of the user $A$, based on two facts that the user $A$ 's public key is not certified and knowledge of the secret value corresponding to the signer's public key is not, even implicitly, checked in the signature verification phase.

To prevent this attack, therefore, the signature verification phase is required to demonstrate that the signer has knowledge of the secret value corresponding to the public key 4 . One instance to provide it is to modify the public key of a user $A$ to include an additional value $x_{A} P_{0}$, where $x_{A}$ is the secret value of the user $A$ and then to add the public key validity check equation

$$
e\left(P_{0}, P_{A}\right)=e\left(P, x_{A} P_{0}\right)
$$

to the signature verification phase 4. This equation basically ensures that the signer $A$ 's public key $\langle X, Y\rangle$ holds the relation $Y=s X$ where $Y=x_{A} P_{0}$ and $X=P_{A}$. Furthermore, it makes sure that the secret value $x_{A}$, chosen by the signer $A$, has been used correctly to obtain $S_{A}=x_{A} Q_{A}+D_{A} 67$. Though an adversary is able to replace the public key $P_{A}$ by $P_{A}^{\prime}$, it is impossible to pass the equation (1) without knowledge of the discrete logarithm of $P_{A}^{\prime}$. Unfortunately, this modification requires four pairing computations though only two are needed per signature if multiple signatures by the same signer are to be verified. Note that this modification only provides a way to defend our attack, and so does not guarantee the security against other attacks [8]. We provide such an example in the following subsection.

Remark 1. Independently, the same attack for the YHG scheme was proposed by Zhang and Feng [16, after we published a preliminary version of this paper 14. While our attack chooses a random exponent $r \in \mathbb{Z}_{q}^{*}$ to construct $U$, they choose $U \in \mathbb{G}_{1}$ itself as a random factor of a forged signature $\sigma=(U, V)$. Since $V=$ $(r+h) x Q_{A}=x\left(U+h Q_{A}\right)$, there is nothing to differentiate between two attacks.

\subsection{Malicious-but-Passive KGC Attack}

Although the above key replacement attack can be prevented by additional checking process, the following malicious-but-passive KGC attack is still applied to the (modified) YHG scheme. Note that this attack is not captured in [15] 
because the security of the YHG scheme is only considered in the original security model of [1], but not of [2].

At first, fix a target user $A$ with the identity $\mathrm{ID}_{A}$. Then the malicious KGC randomly chooses $\alpha \in \mathbb{Z}_{q}^{*}$ and computes $P=\alpha H\left(\mathrm{ID}_{A}\right)$. Then the user $A$ computes his/her public key and private key pair as follows:

$$
\begin{aligned}
& P_{A}=x_{A} P=x_{A} \alpha H\left(\operatorname{ID}_{A}\right) \\
& S_{A}=x_{A} Q_{A}+D_{A}=x_{A}(1 / \alpha) P+D_{A}=(1 / \alpha) P_{A}+D_{A} .
\end{aligned}
$$

Since the KGC knows $\alpha, P_{A}$ and $D_{A}$, the private key $S_{A}$ of the user $A$ can be easily computed by the malicious KGC. As a result, we show that the (modified) YHG scheme is weak against the malicious-but-passive KGC attack though the scheme does not have the same key generation procedure as that of [1].

Remark 2. The Gorantla-Saxena scheme [5] and its improved scheme [17] have a similar structure with the YHG scheme. So, it is very easy to check that both schemes are also vulnerable to a malicious-but-passive KGC attack.

Besides 119], it was shown that certificateless signature schemes in 12 and 13 are insecure against the malicious-but-passive KGC attack [8]. Additionally, the certificateless designated verifier signature scheme proposed by Huang et al. is vulnerable to the malicious-but-passive KGC attack as they follow the same system parameters and user key generation procedure as that of [1.

It can be easily checked that the mediated certificateless signature scheme proposed by Ju et al. [11 is vulnerable to key replacement and malicious-butpassive attacks. A mediated certificateless signature scheme uses an online semitrusted entity called the Security Mediator(SEM) for easy revocation of the user signing key. Since a key replacement attack is not related to the partial private key generated by the KGC (and shared by the user and the SEM), the attack in 9] is applied to the Ju et al.'s scheme directly. Similarly, a malicious-butpassive KGC attack in [2] also works because the SEM does not participate in the system parameters generation phase.

\section{Conclusion}

We showed that the YHG certificateless signature scheme is vulnerable to a key replacement attack and a malicious-but-passive attack, respectively. In addition, we pointed out the same weakness of several certificateless signature schemes under these attacks.

\section{References}

1. Al-Riyami, S.S., Paterson, K.G.: Certificateless public key cryptography. In: Laih, C.-S. (ed.) ASIACRYPT 2003. LNCS, vol. 2894, pp. 452-473. Springer, Heidelberg (2003)

2. Au, M.H., Chen, J., Liu, J.K., Mu, Y., Wong, D.S., Yang, G.: Malicious KGC attacks in certificateless cryptography. In: Proc. of ASIACCS 2007, pp. 302-311 (2007) 
3. Boldyreva, A.: Efficient threshold signature, multisignature and blind signature schemes based on the gap-Diffie-Hellman-group signature scheme. In: Desmedt, Y.G. (ed.) PKC 2003. LNCS, vol. 2567, pp. 31-46. Springer, Heidelberg (2002)

4. Cao, X., Paterson, K.G., Kou, W.: An attack on a certificateless signature scheme. Cryptology ePrint Archive, Report 2006/367

5. Gorantla, M.C., Saxena, A.: An efficient certificateless signature scheme. In: Hao, Y., Liu, J., Wang, Y.-P., Cheung, Y.-m., Yin, H., Jiao, L., Ma, J., Jiao, Y.-C. (eds.) CIS 2005. LNCS (LNAI), vol. 3802, pp. 110-116. Springer, Heidelberg (2005)

6. Hu, B.C., Wong, D.S., Zhang, Z., Deng, X.: Key replacement attack against a generic construction of certificateless signature. In: Batten, L.M., Safavi-Naini, R. (eds.) ACISP 2006. LNCS, vol. 4058, pp. 235-246. Springer, Heidelberg (2006)

7. Hu, B.C., Wong, D.S., Zhang, Z., Deng, X.: Certificateless signature: A new security model and an improved generic construction. Des. Codes. Crypt. 42, 109-126 (2007)

8. Huang, X., Mu, Y., Susilo, W., Wong, D.S., Wu, W.: Certificateless signature revisited. In: Pieprzyk, J., Ghodosi, H., Dawson, E. (eds.) ACISP 2007. LNCS, vol. 4586, Springer, Heidelberg (2007)

9. Huang, X., Susilo, W., Mu, Y., Zhang, F.: On the security of certificateless signature schemes from Asiacrypt 2003. In: Desmedt, Y.G., Wang, H., Mu, Y., Li, Y. (eds.) CANS 2005. LNCS, vol. 3810, pp. 13-25. Springer, Heidelberg (2005)

10. Huang, X., Susilo, W., Mu, Y., Zhang, F.: Certificateless designated verifier signature schemes. In: Proc. of AINA 2006, pp. 15-19 (2006)

11. Ju, H.S., Kim, D.Y., Lee, D.H., Lim, J., Chun, K.: Efficient revocation of security capability in certificateless public key cryptography. In: Khosla, R., Howlett, R.J., Jain, L.C. (eds.) KES 2005. LNCS (LNAI), vol. 3681, pp. 453-459. Springer, Heidelberg (2005)

12. Li, X., Chen, K., Sun, L.: Certificateless signature and proxy signature schemes from bilinear pairings. Lithuanian Mathematical Journal 45, 76-83 (2005)

13. Liu, J.K., Au, M.H., Susilo, W.: Self-generated-certificate public key cryptography and certificateless signature/encryption scheme in the standard model. In: Proc. of ASIACCS 2007, pp. 273-283 (2007)

14. Park, J.H.: An attack on the certificateless signature scheme from EUC Workshops 2006. Cryptology ePrint Archive, Report 2006/442

15. Yap, W.-S., Heng, S.-H., Goi, B.-M.: An efficient certificateless signature scheme. In: Zhou, X., Sokolsky, O., Yan, L., Jung, E.-S., Shao, Z., Mu, Y., Lee, D.C., Kim, D., Jeong, Y.-S., Xu, C.-Z. (eds.) EUC 2006. LNCS, vol. 4097, pp. 322-331. Springer, Heidelberg (2006)

16. Zhang, Z., Feng, D.: Key replacement attack on a certificateless signature scheme. Cryptology ePrint Archive, Report 2006/453

17. Zhang, J., Mao, J.: Security analysis of two signature schemes and their improved schemes. In: Gervasi, O., Gavrilova, O. (eds.) ICCSA 2007. Part I. LNCS, vol. 4705, pp. 589-602. Springer, Heidelberg (2007)

18. Zhang, Z., Wong, D.S., Xu, J., Feng, D.: Certificateless public-key signature: Security model and efficient construction. In: Zhou, J., Yung, M., Bao, F. (eds.) ACNS 2006. LNCS, vol. 3989, pp. 293-308. Springer, Heidelberg (2006) 